\title{
EVALUACIÓN DE LA MEMORIA SEMÁNTICA: ESTUDIO TRANSVERSAL EN PERSONAS SANAS Y EN PACIENTES CON ENFERMEDAD DE ALZHEIMER
}

\author{
Luis A. SAÚl, M. Ángeles LÓPEZ-GONZÁLEZ, FERNANDO RuBiO-GARAY \\ Y MiguEL A. CARRASCO
}

Facultad de Psicología, Universidad Nacional de Educación a Distancia, Madrid

\begin{abstract}
Resumen: En el presente trabajo se estudia la evolución y el deterioro de la memoria semántica en personas sanas y con demencia tipo Alzheimer. Mediante una prueba de fluencia categorial semántica, se evaluó la frecuencia de producción de ejemplares de cuatro categorías semánticas básicas pertenecientes a los dominios Seres Vivos (SV) y Seres No Vivos (SNV). La muestra está constituida por 80 participantes clasificados en cuatro grupos: niños, adultos jóvenes, ancianos sanos y ancianos con enfermedad de Alzheimer. Los resultados mostraron una mayor producción de los adultos jóvenes en los dominios SV y SNV, seguido de los niños y los ancianos sanos y, por último, los ancianos con Enfermedad de Alzheimer. En cuanto al sexo, las mujeres tuvieron una mejor ejecución pero solo en la muestra de personas sanas. Además, se computó el número de errores, siendo el grupo de niños el que mayor número cometió debido fundamentalmente a la inclusión de ejemplares no pertinentes (sobregeneralización).
\end{abstract}

Palabras Clave: Categorización, memoria semántica, fluidez verbal, enfermedad de Alzheimer, estudio transversal.

\section{Evaluation of semantic memory: A cross-sectional study in healthy people and comparison with Alzheimer's disease patients}

\begin{abstract}
The present paper evaluates the evolution and deterioration of semantic memory in a healthy population and in people with Alzheimer's disease. Using a categorical semantic fluency test, we evaluated the frequency of production of copies of four basic semantic categories belonging to the domains Living Things (LT) and Non-Living things (NLT). The sample consisted of 80 subjects classified in four groups: children, young adults, healthy elderly persons and elderly people with Alzheimer's disease. The results showed a greater production by young adults in the domains LT and NLT, followed by children and the elderly healthy and, finally, the elderly Alzheimer's disease patients. Regarding gender, females showed a better performance but only in the sample of healthy people. Furthermore, the number of errors was considered: the group with a higher number of errors was the children's, mainly due to the inclusion of non pertinent elements (overgeneralization).
\end{abstract}

Key words: Categorization, semantic memory, verbal fluency, Alzheimer's disease, cross-sectional study.

\section{INTRODUCCIÓN}

La memoria semántica (MS) o conceptual se refiere al conocimiento general sobre el mun-

Recibido: 25-mayo-2010; aceptado: 2-julio-2010

Correspondencia: Luis A. Saúl, UNED, Departamento de Psicología de la Personalidad, Evaluación y Tratamiento Psicológicos, Facultad de Psicología, Juan del Rosal 10, 28040 Madrid.

Correo-e: lasaul@psi.uned.es do no ligado al contexto espacio-temporal; engloba además el conocimiento de las palabras y de su significado, de los conceptos y relaciones y reglas para su utilización (De Vega, 2007). A través de ella, cualquier información puede ser expresada en términos de conocimiento impersonal puesto que carece de referencias biográficas (Tudela, 1985). El contenido de la MS, al igual que la memoria episódica se refiere a contenidos del tipo «saber qué», frente a 
conocimientos procedimentales del tipo a «saber cómo» (Diges y Perpiña, 2008). Las alteraciones mnemotécnicas son los primeros signos y/o síntomas cognitivos que surgen durante el proceso de envejecimiento, tanto en personas sanas como en los primeros estadios de algunos tipos de demencia, como en la enfermedad de Alzheimer (EA; Díaz y Peraíta, 2008; Fernández-Calvo et al., 2010; Román y Sánchez, 1998). Los cambios que se producen en la MS en el proceso evolutivo normal y patológico de las personas mayores, se manifiestan de manera habitual a través de dos tipos de disociaciones o deterioros categoriales. Por un lado, disociaciones entre el conocimiento semántico de las categorías y el conocimiento de los ejemplares que pertenecen a las mismas (p.ej., Tippett, Mcauliffe y Farah, 1995); y por otro, las disociaciones entre categorías de seres vivos (SV) versus seres no vivos (SNV), en las que se produce un deterioro selectivo en las mismas (deterioro de un dominio categorial). Para investigar la estructura interna y contenido de las categorías o conceptos naturales se han utilizado fundamentalmente dos procedimientos metodológicos: 1) la técnica de producción de ejemplares (fluidez verbal, fluencia categorial semántica o fluidez categorial), en la que los sujetos han de nombrar todos los ejemplares que conozcan de una determinada categoría de nivel supraordenado en un periodo de tiempo concreto (Battig y Montague, 1969; Nelson, 1974; Posnansky, 1978; Puente y Poggioli, 1993; Soto, 1981; Soto, Sebastián, García y del Amo, 1982, 1994); y 2) mediante escalas numéricas que evalúan la pertenencia de un ejemplar a una categoría supraordenada (Rosch, 1975; Uyeda y Mandler, 1980; Soto, 1982). Además se han utilizado otros procedimientos como las tareas de denominación de emparejamiento palabra-dibujo, de clasificación, de conocimiento semántico, de dibujo y de decisión de objetos (ver Moreno, 2006, para una revisión). Por otra parte, para evaluar el deterioro semántico categorial más común (disociación en los dominios SV versus SNV) en pacientes con daño cerebral y con demencias como la EA, se han diseñado instrumentos específicos en español como la batería Evaluación de la Memoria Semántica en Demencia tipo Alzheimer
(EMSDA) (Peraita, González Labra, SánchezBernardos y Galeote, 2000) o la Batería Nombela (Moreno y Cañamón, 2005).

Con el paso del tiempo, en las personas mayores se produce una alteración en el acceso léxico, incrementándose fenómenos lingüísticos como la punta de la lengua (Burke, Mackay, Whorthey y Wade, 1991; Burke, Whorthey y Martin, 1988; Shafto, Burke, Stamatakis, Tamp y Tyler, 2007) aunque no necesariamente se produce una alteración del conocimiento léxico ya que el vocabulario se conserva (Salthouse, 1988; Wingfield, Aberdeen y Stine, 1991) e incluso se puede incrementar (Camarazza, 1990). No obstante, es preciso tener en cuenta que variables como la educación, el cociente intelectual y la salud podrían estar influyendo en los estudios de memoria (Huppert, 1991; Muela, García-León y Jiménez, 2007). Así, diversas investigaciones han puesto de manifiesto la importancia de los años de escolaridad en el rendimiento en tareas de fluencia fonológica (p. ej. Burie, Gramunt, Böhm, Rodés y Peña-Casanova, 2004) y en tareas de fluencia categorial semántica (Auriacombe, Fabrigoule, Lafont, Jacqmin-Gadda y Dartigues, 2001; Moreno, Adrián, Buiza y González, 2004; Ramírez, Ostrosky-Solís, Fernández y Ardilla-Ardilla, 2005; Valencia et al., 2000; Wiederholt, Cahn, Butters, Salmon, Kritz-Siverstein y BarretConnor, 1993).

Además de los problemas de acceso al léxico que se producen durante el envejecimiento normal, la MS se ve gravemente afectada en su estructura y organización en determinadas enfermedades neurodegenerativas como en la demencia semántica o en la demencia tipo Alzheimer (Díaz y Peraíta, 2008; Gutiérrez-Cabello et al., 2007). En esta última, junto a los síntomas iniciales relacionados con la incapacidad para encontrar y recuperar de forma espontánea determinadas palabras y la reducción significativa de la disponibilidad léxica categorial (Peraita et al., 2000), se produce un deterioro progresivo en otras variedades de memorias declarativas como la memoria autobiográfica o la memoria pública (Cuetos, Rodríguez-Ferreiro y Martínez, 2003). El deterioro en la MS se hace evidente por la pobre ejecución de los pacientes con EA en tareas de fluencia catego- 
rial semántica, pues generan un menor número de ejemplares por categoría que las personas sanas (Díaz, Peraita y Garriga, 2000; Peraita y Sánchez-Bernardos, 1998) y producen un mayor número de errores supraordenados pues, a diferencia de las personas sanas y los enfermos con otras demencias, denominan con el nombre de la categoría y no con el nombre del elemento (p.ej., verduras en vez de col o lechuga) (Hodges, Salmon y Butters, 1991; Ober, Dronkers, Koss, Delis y Friedland, 1986). Puesto que los trabajos neuropsicológicos ofrecen resultados contradictorios en cuanto al patrón de deterioro de los dominios Seres Vivos (SV) vs. Seres No Vivos (SNV) (p.ej., Gonnerman, Andersen, Devlin, Kempler y Seidenberg, 1997), conviene analizar si la producción de ejemplares se ve afectada diferencialmente en ambos dominios para profundizar, así, en el estudio de la organización funcional y estructural de la MS.

El objetivo de este trabajo fue evaluar la MS mediante la ejecución de una tarea de fluidez verbal en función de la edad y del sexo tanto en personas sanas como en personas con enfermedad de Alzheimer. En primer lugar, se esperan encontrar diferencias en la producción total de ejemplares y en los dominios SV/SNV en función del sexo y de los grupos establecidos (niños, adultos jóvenes, ancianos sanos y ancianos con EA) de manera que las personas con EA producirán un número menor de ejemplares que el resto de grupos. En segundo lugar, se esperan hallar diferencias en la producción de ejemplares en el conjunto de personas sanas y en pacientes con EA en función del sexo, en el sentido de una mayor producción de ejemplares por parte de las mujeres. Finalmente, es de esperar que el grupo de los niños cometa un mayor número de errores en la producción total de ejemplares.

\section{MÉTODO}

\section{Participantes}

En el presente estudio, participaron un total de 80 personas que se dividieron en cuatro grupos: niños de 10 años (10 niños y 10 niñas; $M$
$=10 ; D T=0,00)$; adultos jóvenes de entre $18 \mathrm{y}$ 30 años (10 hombres y 10 mujeres; $M=25,25$; $D T=4,22)$; ancianos sanos, con un rango de edad de entre 65 y 86 años (10 ancianos y 10 ancianas; $M=74,45 ; D T=6,25)$; y ancianos con EA de entre 70 y 85 años (10 ancianos y 10 ancianas; $M=78,15 ; D T=4,18$ ). La muestra se obtuvo a través de un procedimiento de muestreo no probabilístico accidental. El grupo de niños procedía de un colegio en el que cursaban $5^{\circ}$ de Educación Primaria; en todos ellos, el desarrollo psicomotor era acorde con la edad cronológica, a juicio de los educadores. La muestra del grupo de adultos jóvenes se extrajo de un Instituto de Formación de Empleo y pertenecían a la misma área sociocultural. El grupo de ancianos sanos procedía de una Comunidad Benedictina en régimen de clausura, en el caso de las mujeres, y de una Residencia de Mayores cercana al convento, en el caso de los hombres. El grupo de las personas con EA fueron reclutadas en un centro de día para personas con Alzheimer. Para la selección de la muestra, se tuvieron en cuenta como criterios de inclusión dos variables sociodemográficas: el nivel de estudios (todos ellos con estudios primarios) $\mathrm{y}$ el nivel socioeconómico y cultural, que se consideró promedio y representativo de la ciudad donde se realizó este estudio. El único criterio de exclusión fue que los participantes no padecieran ningún tipo de trastorno psiquiátrico o enfermedad médica o neurológica (a excepción de la EA) que pudiera interferir en la realización de la tarea.

\section{Instrumentos}

Para evaluar la capacidad de los participantes en la producción de ejemplares de categorías supraordenadas en un tiempo determinado, se elaboraron unas hojas de registro en las que se señalaban cuatro categorías: dos de origen biológico (mamíferos y árboles) y las otras dos, de origen objetal (electrodomésticos y oficios). En esta ficha, se recogieron también variables sociodemográficas (edad, sexo y presencia/ausencia de enfermedades), así como datos específicos de la producción de ejemplares: número de ejemplares por categoría, errores y repeticiones. 


\section{Procedimiento}

Los participantes fueron evaluados en sus contextos naturales. Se les explicó en qué consistía el estudio y se les entregaron las hojas de registro (salvo al grupo de ancianos con EA) en las que debían anotar: sexo, estado civil, estudios realizados y enfermedades que padecían). La tarea de los participantes consistía en producir oralmente, en un tiempo máximo de dos minutos por categoría, todos los tipos/ejemplares básicos pertenecientes a las categorías semánticas propuestas. Para controlar la reactividad a la situación experimental y al proceso de evaluación y con el fin de mejorar la validez ecológica, se integró dentro de la situación experimental lo cotidiano y lo habitual del comportamiento de los sujetos. Así, personas cercanas a las mismas (como la maestra en el caso de los niños, la abadesa en el caso del convento de clausura, y los educadores en el caso de las personas con EA) eran las encargadas de realizar la prueba, en un intento de conseguir unas condiciones lo más naturales posibles. Estos entrevistadores entrenados nombraban cada una de las categorías $\mathrm{y}$, a continuación, el sujeto debía generar tantos ejemplares como conociera de dicha categoría, en el mismo orden en que los iba recordando. Se anotaron los ejemplares en una hoja, respetando el orden de producción. Se recogieron todos los ejemplares producidos aunque no fueran miem- bros de la categoría donde se incluyeron. El singular y el plural y los sinónimos se consideraron como el mismo ítem; lo mismo que el género cuando derivaba del mismo sema. Por el contrario, cuando el masculino y el femenino de las palabras procedían de distinta raíz léxica, se consideraron entradas diferentes. Las categorías subordinadas o supraordenadas (p.ej., túrmix o electrodoméstico) se consideraron errores.

\section{Diseño}

Se trata de un diseño ex post facto prospectivo evolutivo transversal, en el que se controla la variable nivel de estudios. La variable dependiente o criterio fue la fluidez verbal, operativizada mediante el número de ejemplares producidos por unidad de tiempo (frecuencia de ejemplares) atendiendo al tipo de domino (SV/SNV) o a las categorías semánticas supraordenadas (electrodomésticos, oficios, mamíferos y árboles). Las variables independientes o predictoras fueron el sexo (hombres vs. mujeres), edad (niños, adultos jóvenes, ancianos) y presencia o no de demencia (ancianos sanos vs. ancianos con EA).

\section{RESULTADOS}

En primer lugar, se realizaron análisis descriptivos de las categorías analizadas en función del

Tabla 1. Medias $(D T)$ en la producción de ejemplares (aciertos) para cuatro categorías semánticas: electrodomésticos, oficios, mamíferos y árboles)

\begin{tabular}{|c|c|c|c|c|c|c|c|c|}
\hline & \multicolumn{2}{|c|}{ Electrodomésticos } & \multicolumn{2}{|c|}{ Oficios } & \multicolumn{2}{|c|}{ Mamíferos } & \multicolumn{2}{|c|}{ Árboles } \\
\hline & Hombres & Mujeres & Hombres & Mujeres & Hombres & Mujeres & Hombres & Mujeres \\
\hline & $M(D T)$ & $M(D T)$ & $M(D T)$ & $M(D T)$ & $M(D T)$ & $M(D T)$ & $M(D T)$ & $M(D T)$ \\
\hline Niños & $\begin{array}{l}10,70 \\
(3,12)\end{array}$ & $\begin{array}{l}10,50 \\
(3,50)\end{array}$ & $\begin{array}{l}10,50 \\
(3,59)\end{array}$ & $\begin{array}{l}11,50 \\
(3,56)\end{array}$ & $\begin{array}{l}10,60 \\
(3,74)\end{array}$ & $\begin{array}{c}8,50 \\
(4,47)\end{array}$ & $\begin{array}{c}8,50 \\
(1,26)\end{array}$ & $\begin{array}{c}9,20 \\
(1,68)\end{array}$ \\
\hline Adultos jóvenes & $\begin{array}{l}13,90 \\
(3,84)\end{array}$ & $\begin{array}{l}15,40 \\
(3,89)\end{array}$ & $\begin{array}{l}16,60 \\
(3,74)\end{array}$ & $\begin{array}{l}18,90 \\
(6,55)\end{array}$ & $\begin{array}{l}10,00 \\
(5,55)\end{array}$ & $\begin{array}{c}8,50 \\
(4,19)\end{array}$ & $\begin{array}{l}11,50 \\
(5,06)\end{array}$ & $\begin{array}{l}13,00 \\
(3,33)\end{array}$ \\
\hline Ancianos sanos & $\begin{array}{c}5,20 \\
(3,08)\end{array}$ & $\begin{array}{l}10,20 \\
(5,07)\end{array}$ & $\begin{array}{c}7,80 \\
(3,19)\end{array}$ & $\begin{array}{l}14,60 \\
(6,58)\end{array}$ & $\begin{array}{c}6,50 \\
(4,30)\end{array}$ & $\begin{array}{l}11,70 \\
(6,00)\end{array}$ & $\begin{array}{c}7,00 \\
(2,78)\end{array}$ & $\begin{array}{l}13,00 \\
(4,54)\end{array}$ \\
\hline Ancianos con EA & $\begin{array}{c}2,10 \\
(1,91)\end{array}$ & $\begin{array}{c}2,30 \\
(2,05)\end{array}$ & $\begin{array}{c}3,50 \\
(2,12)\end{array}$ & $\begin{array}{c}1,20 \\
(1,47)\end{array}$ & $\begin{array}{c}4,00 \\
(2,78)\end{array}$ & $\begin{array}{c}1,40 \\
(1,64)\end{array}$ & $\begin{array}{c}2,80 \\
(2,44)\end{array}$ & $\begin{array}{c}1,10 \\
(1,52)\end{array}$ \\
\hline
\end{tabular}


Tabla 2. Índices de variabilidad léxica para los cuatro grupos e índices de variabilidad total

\begin{tabular}{|c|c|c|c|c|c|c|c|c|c|}
\hline \multirow{2}{*}{ Grupos } & \multicolumn{3}{|c|}{ Seres Vivos } & \multicolumn{3}{|c|}{ Seres No Vivos } & \multicolumn{3}{|c|}{ Producción Total } \\
\hline & Casos & Tipos & $I V L$ & Casos & Tipos & $I V L$ & Casos & Tipos & $I V L$ \\
\hline Niños & 367 & 69 & 0,19 & 427 & 157 & 0,37 & 794 & 226 & 0,28 \\
\hline Adultos Jóvenes & 430 & 110 & 0,26 & 633 & 248 & 0,39 & 1063 & 358 & 0,34 \\
\hline Ancianos Sanos & 381 & 114 & 0,30 & 377 & 152 & 0,40 & 758 & 266 & 0,35 \\
\hline Ancianos con EA & 98 & 43 & 0,44 & 97 & 52 & 0,54 & 195 & 95 & 0,49 \\
\hline Suma total & 1259 & 170 & 0,13 & 1534 & 368 & 0,23 & 2793 & 538 & 0,19 \\
\hline
\end{tabular}

Nota $. \mathrm{IVL}=$ índice de variabilidad léxica.

Tabla 3. Medias (DT) de los dominios SV/SNV y producción total (aciertos) en función de los cuatro grupos definidos (niños, adultos jóvenes, ancianos sanos y ancianos con EA) y en función del sexo (hombres y mujeres)

\begin{tabular}{|c|c|c|c|c|c|c|c|c|c|}
\hline & & \multicolumn{4}{|c|}{ Grupos } & \multicolumn{3}{|c|}{ Sexo } & \multirow{3}{*}{$\begin{array}{c}\begin{array}{c}\text { Interacción } \\
\text { grupos } \times \text { sexo }\end{array} \\
F_{(3,632)}\end{array}$} \\
\hline & Niños & $\begin{array}{l}\text { Adultos } \\
\text { jóvenes }\end{array}$ & $\begin{array}{l}\text { Ancianos } \\
\text { sanos }\end{array}$ & $\begin{array}{c}\text { Ancianos } \\
\text { con EA }\end{array}$ & & Hombres & Mujeres & & \\
\hline & $M(D T)$ & $M(D T)$ & $M(D T)$ & $M(D T)$ & $F_{(3,632)}$ & $M(D T)$ & $M(D T)$ & $F_{(1,632)}$ & \\
\hline Seres vivos & $\begin{array}{l}18,40^{a} \\
(5,21)\end{array}$ & $\begin{array}{c}21,50^{a} \\
(7,22)\end{array}$ & $\begin{array}{l}19,10^{\mathrm{a}} \\
(10,05)\end{array}$ & $\begin{array}{c}4,65^{b} \\
(4,35)\end{array}$ & $27,35 * * *$ & $\begin{array}{c}15,23 \\
(8,23)\end{array}$ & $\begin{array}{c}16,60 \\
(10,85)\end{array}$ & 0,89 & $5,42 * *$ \\
\hline Seres No vivos & $\begin{array}{c}21,60^{\mathrm{a}} \\
(5,08)\end{array}$ & $\begin{array}{c}32,40^{b} \\
(8,54)\end{array}$ & $\begin{array}{l}18,90^{\mathrm{a}} \\
(10,26)\end{array}$ & $\begin{array}{l}4,55^{c} \\
(3,50)\end{array}$ & $57,07 * * *$ & $\begin{array}{c}17,58 \mathrm{a} \\
(10,71)\end{array}$ & $\begin{array}{c}21,15 b \\
(13,65)\end{array}$ & $5,54^{*}$ & $3,89^{*}$ \\
\hline Producción total & $\begin{array}{c}40,00^{\mathrm{a}} \\
(9,78)\end{array}$ & $\begin{array}{c}53,90^{\mathrm{b}} \\
(13,77)\end{array}$ & $\begin{array}{c}38,00^{\mathrm{a}} \\
(19,87)\end{array}$ & $\begin{array}{c}9,20^{\mathrm{c}} \\
(7,45)\end{array}$ & $45,84 * * *$ & $\begin{array}{c}32,80 \\
(18,16)\end{array}$ & $\begin{array}{c}37,75 \\
(23,62)\end{array}$ & 3,18 & $5,28 * *$ \\
\hline
\end{tabular}

Nota: Los grupos cuyas medias comparten superíndice significa que no son significativamente diferentes entre sí en las comparaciones post-hoc. $* p<0,05 ; * * p<0,01 ; * * * p<0,001$.

sexo y de los grupos definidos (ver Tabla 1). Todos los análisis estadísticos fueron realizados utilizando el programa informático SPSS, versión 17 (2008). Además se halló el índice de variabilidad léxica (IVL), que resulta de la división de los tipos entre los casos u ocurrencias. Los tipos se corresponden al total de ejemplares diferentes y las ocurrencias al número total de ejemplares producidos (véase la Tabla 2).

\section{Producción de ejemplares en los dominios} SV/SNV y producción total en función de los grupos y del sexo.

Se realizó un ANOVA bifactorial con interacción (contraste bilateral) de 4 (grupos) $\times 2$ (sexo) para la variable dependiente producción total de ejemplares (véase la Tabla 3). Los resultados mostraron diferencias significativas para el efecto de interacción $(p<0,05)$. Las comparaciones post hoc para la variable grupos se realizaron mediante el procedimiento de Tukey, asumiendo la homogeneidad de las varianza (test de Levene, $p>0,05$ ).

El análisis de la producción de ejemplares en los dominios SV/SNV se realizó mediante dos análisis de la varianza (ANOVA) bifactoriales con interacción (contraste bilateral), tomando como variables independientes el grupo de edad (con cuatro niveles) y el sexo (con dos niveles). Los resultados mostraron diferencias significativas para el efecto de interacción sexo y grupo de edad $(p<0,01)$ en el dominio de los 
SV. Puesto que existe homogeneidad en la varianza (test de Levene, $p>0,05$ ), las comparaciones múltiples se realizaron mediante la prueba de Tukey (véase la Tabla 3). En el dominio de los SNV se encontraron diferencias significativas en la interacción sexo con grupo de edad $(p<0,05)$. Debido a que la varianza no fue homogénea (test de Levene, $p<0,05$ ), las comparaciones múltiples se realizaron mediante la prueba Games-Howell (véase la Tabla 3).

Producción de ejemplares en personas sanas $y$ en personas con EA en función del sexo

Los resultados del ANOVA de un factor (hombres vs mujeres) para la variable dependiente producción total de ejemplares en personas sanas mostraron diferencias estadísticamente significativas $(F(1,58)=4,48 ; p<0,05)$ entre hombres $(M=39,6 ; D T=15,27)$ y mujeres $(M=48,33 ; D T=16,26)$. Respecto a la muestra de ancianos sanos, en la prueba $U$ de Mann-Whitney, (contraste bilateral) se encontraron diferencias estadísticamente significativas en relación al sexo, tanto en producción total $(U=17,00, Z=-2,50 ; p<0,05)$, como en los dominios de SV $(U=15,50, Z=-2,61 ; p<$ $0,05)$ y $\operatorname{SNV}(U=19,50, Z=-2,31 ; p<0,05)$, a favor de las mujeres. En cuanto al grupo de personas con EA, en la prueba U de MannWhitney (contraste bilateral), la puntuación media de los hombres y de las mujeres no difirió significativamente en cuanto a la producción total $(U=26,00, Z=-1,81$; n.s. $)$ y al dominio de $\operatorname{SNV}(U=31,50, Z=-1,41$; n.s. $)$; sin embargo, en el dominio de los SV se encontraron diferencias estadísticamente significativas a favor de los hombres $(U=21,00, Z=-2,21$; $p<0,05)$.

\section{Producción total de ejemplares erróneos en función del sexo y el grupo de edad}

Para el análisis de la producción total de ejemplares erróneos se realizó un ANOVA bifactorial (contraste bilateral), de 4 (grupos) $\times 2$ (sexo). No resultaron significativos los efectos de ninguno de los dos factores, ni tampoco de la interacción entre ambos. Los resultados se muestran en la Tabla 4.

\section{DISCUSIÓN}

En el presente estudio, mediante una metodología cuantitativa, se han investigado las diferencias de la MS en distintos grupos de edad en personas sanas, así como el deterioro de la misma en personas con demencia senil tipo Alzheimer. La evaluación de dichos aspectos se ha efectuado a través de una tarea de fluencia categorial semántica (fluidez semántica categorial o fluidez verbal), llevada a cabo por una muestra de personas de diferentes edades: niños, adultos jóvenes, ancianos sanos y ancianos con EA, todos ellos con un mismo nivel de escolaridad (estudios primarios).

Los resultados encontrados confirman la primera hipótesis planteada, al constatar la pobre ejecución de los ancianos con EA en tareas cognitivas de fluidez verbal. Dichos resultados se han mostrado en consonancia con diversas

Tabla 4. Medias (DT) de la producción total de errores en función de los cuatro grupos definidos (niños, adultos jóvenes, ancianos sanos y ancianos con EA) y en función del sexo (hombres y mujeres)

\begin{tabular}{|c|c|c|c|c|c|c|c|c|c|}
\hline & \multicolumn{4}{|c|}{ Grupos } & \multicolumn{3}{|c|}{ Sexo } & \multicolumn{2}{|r|}{$\begin{array}{c}\text { Interacción } \\
\text { grupos } \times \text { sexo }\end{array}$} \\
\hline & Niños & $\begin{array}{l}\text { Adultos } \\
\text { jóvenes }\end{array}$ & $\begin{array}{l}\text { Ancianos } \\
\text { sanos }\end{array}$ & $\begin{array}{l}\text { Ancianos } \\
\text { con EA }\end{array}$ & & Hombres & Mujeres & & \\
\hline & $M(D T)$ & $M(D T)$ & $M(D T)$ & $M(D T)$ & $F_{(3,632)}$ & $M(D T)$ & $M(D T)$ & $F_{(1,632)}$ & $F_{(3,632)}$ \\
\hline $\begin{array}{l}\text { Producción total } \\
\text { de errores }\end{array}$ & $\begin{array}{c}1,40 \\
(1,04)\end{array}$ & $\begin{array}{c}0,75 \\
(0,91)\end{array}$ & $\begin{array}{c}0,55 \\
(1,60)\end{array}$ & $\begin{array}{c}0,90 \\
(1,33)\end{array}$ & 1,62 n.s. & $\begin{array}{c}1,07 \\
(1,43)\end{array}$ & $\begin{array}{c}0,72 \\
(1,06)\end{array}$ & $\begin{array}{l}1,51 \\
\text { n.s. }\end{array}$ & 0,09 n.s. \\
\hline
\end{tabular}


investigaciones realizadas en este ámbito (ver meta-análisis de Henry, Crawford y Phillips, 2004), tanto en el dominio de SV como en el de SNV; así como en aquellos trabajos que han comparado personas sanas mayores (Díaz et al., 2000; Peraita y Sánchez-Bernardos, 1998; Sebastián y Elosúa, 2002) y jóvenes (Galeote y Peraita, 1999). A pesar de que los ancianos con EA presentan una menor producción de ejemplares (casos u ocurrencias), es el grupo que muestra un índice de variabilidad léxica (IVL) más alto, superior incluso al de los ancianos sanos. En cuanto a los niños, la producción de ejemplares fue similar a la de los ancianos sanos; aunque se observa que los ejemplares producidos por los niños están circunscritos a unos pocos tipos. Este último aspecto es relevante, por cuanto que la comparación entre niños y ancianos no suele aparecer en la literatura, aunque sí se han realizado estudios que comparan grupos de adultos jóvenes y niños (Piñeiro, Morenza, Torres y Sierra, 1999) o que evalúan la producción de categorías semánticas exclusivamente en niños (Koren, Kofman y Berger, 2005; Torres, Piñeiro, Morenza e Inguanzo, 2000) o en adultos jóvenes (Buriel, Gramunt, Böhm, Rodés, y Peña-Casanova, 2004). Diversas investigaciones han revelado un incremento de la fluidez verbal desde la niñez (p. ej., Koren et al., 2005; Sauzeon, Lestage, Raboutet, N' Kaoua y Claverie, 2004) hasta la madurez (Lara-Useche, Pineda, Henao-Arboleda, ArboledaRamírez, Aguirre-Acevedo y Lopera, 2006), en consonancia con nuestro estudio, en el que aparece una mayor fluencia categorial semántica en el grupo de los jóvenes. Sin embargo, es difícil precisar a partir de qué momento comienza a producirse el declive en la MS o si, como afirman otros autores (véase Román y Sánchez, 1998), durante el envejecimiento se produce un deterioro en la memoria episódica más que en la semántica. Los hallazgos encontrados en ancianos sanos podrían deberse a un proceso de evolución natural de la MS. Como refiere Juncos (1998), todo indica que las personas mayores sanas responden más a un problema de ejecución que de competencia. Así pues, aunque el conocimiento se mantiene, tanto desde el punto de vista conceptual, como semántico y fonológico, se observan ciertas dificultades para acceder a este conocimiento, de manera que hay una incapacidad para encontrar los nombres, se incrementan los tiempos de reacción y se usan paráfrasis como estrategias compensatorias (Juncos et al., 1998); es decir, en las personas mayores, los problemas de acceso al léxico serían consecuencia más bien de un déficit de memoria operativa y de capacidad atencional (Buiza, 2001) que de competencia.

Con relación a la segunda hipótesis planteada, tal y como esperábamos, los resultados sobre el efecto del sexo en las personas ancianas sanas indican una mayor producción de categorías semánticas por parte de las mujeres frente a los hombres, tanto en SV como en SNV y en producción total. Los presentes resultados son congruentes con aquellos trabajos que indican una cierta ventaja de las mujeres con respecto a los hombres en tareas de fluencia verbal (Gordon y Lee, 1986; Stumpf, 1995; Pineda, Galeano, Giraldo, 1995). En cambio, cuando se analiza la producción en la muestra de EA, los resultados no muestran diferencias entre hombres y mujeres, en la línea de algunas investigaciones (Galeote y Peraita, 1999) aunque sí se observa un patrón de producción mayor en hombres que en mujeres, aunque no alcanza la significación estadística. En cambio, si solo se tiene en cuenta la muestra de personas sanas, la producción total de aciertos es mayor en las mujeres que en los hombres. En relación a la dicotomía SV/SNV, encontramos diferencias estadísticamente significativas en el dominio de SNV a favor de las mujeres y una tendencia a una mayor producción de los SV también en ellas. Sin embargo, la literatura refleja ventajas de las mujeres en las categorías de los dominios de los SV y de los hombres en el dominio de los SNV (Barbarotto, Laiacona, Macchi y Capitani, 2002; Capitani, Laiacona y Barbarotto, 1999; Laws, 2004; Moreno, Rodríguez y Peraíta, 2007). No obstante, para algunos autores (Marra, Ferraccioli y Gianotti, 2007) las desigualdades encontradas en las categorías son debidas principalmente a la familiaridad de los participantes con las categorías más que a la existencia de diferencias innatas en relación al sexo. Sin embargo, también podrían deberse a la falta de control en muchos de los trabajos de una de las variables que influyen en el rendi- 
miento en tareas de fluencia categorial semántica, como es el nivel de estudios. Es conocido (p. ej. Buriel et al., 2004) que las personas con una mayor escolarización, rinden mejor en tareas de fluencia categorial semántica (Auriacombe et al., 1998; Fillembaum et al., 2001; Moreno et al., 2004; Ramírez, et al., 2005; Valencia et al. 2000; Wiederholt et al., 1993). Algunos trabajos informan que el nivel de estudios predice hasta el $25 \%$ de la varianza de la producción total de ejemplares (Gladsjo, Schuman, Evans, Peavy, Miller y Heaton, 1999). Es por esto que, con el objeto de controlar la variable escolarización, en esta investigación se seleccionaron sólo sujetos con estudios primarios. En este estudio, también hay que tener presente que el efecto diferenciador de la fluencia semántica en el caso de las mujeres ancianas, (las monjas de clausura), podría resultar del grado de familiaridad o incluso del grado de conocimiento y experiencia (grado de experticia) que tienen estas personas en algunas categorías (p.ej. árboles). Así, distintos grados de conocimiento sobre una categoría determinada pueden provocar diferentes representaciones jerárquico-conceptuales, lo que confirmaría la dificultad de establecer comparaciones entre los distintos grupos de estudio. Con respecto a la dicotomía SV/SNV, el grupo de Alzheimer presenta un deterioro similar, dentro de su gran pobreza productiva y, por tanto, no se confirma una mayor pérdida en la categoría SV frente a la SNV, en la línea de algunos trabajos (Galeote y Peraita, 1999), pero contrario a otras investigaciones (Moreno-Rodríguez y Peraita, 2007) que encuentran un deterioro más severo en SV. Esta similitud en los patrones de producción de ejemplares generados también se observa en ancianos sanos (p.ej. Peraita y Moreno, 2006).

Finalmente, en relación con la tercera hipótesis propuesta, los resultados muestran que los niños cometen el mayor número de errores, lo que apoya la hipótesis planteada. La mayoría de ellos se debieron a la inclusión de ejemplares subordinados (p.ej., game boy, play station...) y al fenómeno de sobregeneralización o inclusión de ejemplares no pertinentes (Mervis, Catlin y Rosch, 1975; Neimark, 1974; Nelson, 1974). Esta sobregeneralización no ocurre por igual en todas las categorías estudiadas ya que sólo se observa en mamíferos, lo cual podría ser indicativo de que el desarrollo de las categorías no es homogéneo y unas son adquiridas antes que otras, en ocasiones relacionado con la interacción del niño con los ejemplares. Este fenómeno podría deberse a diferentes razones, entre las que podríamos mencionar: la escasa estructura de las categorías infantiles (Piñeiro et al., 1999); a que los niños generan ejemplares que ningún adulto consideraría como pertinente $\mathrm{y}$, por el contrario, no incluyen otros que los adultos sí consideran en sus categorías (Rosner y Hayes, 1977); el análisis diferencial que hacen adultos y niños de los atributos y propiedades de los objetos (Mervis y Greco, 1984; Puente y Pogglioli, 1993); a la incompleta comprensión que tienen del significado de las palabras (Miller y Gildea, 1987); y a la inadecuada selección que hacen los niños de los ejemplares que cumplen con los requisitos de la tarea antes de producirlos.

En cuanto a las personas con EA, cometen más errores que los ancianos sanos y los jóvenes; las equivocaciones más habituales son debidas a la inclusión de ejemplares de un nivel supraordenado (p.ej., «electrodoméstico» como ejemplo de electrodoméstico) pero podrían deberse más que a un error a la repetición de palabras (ecolalia), consecuencia de la propia evolución de la enfermedad. La mayor comisión de errores de los ancianos con EA frente a un grupo de controles sanos se encuentra en la línea de algunos trabajos (Cuetos et al., 2003; Hodges et al., 1991) que han mostrado que los pacientes con EA presentan dificultades en la MS de tipo anómico desde estadíos tempranos de la enfermedad, sobre todo debidas a la pérdida gradual del significado de las palabras.

En este trabajo, se han planteado algunas limitaciones de carácter metodológico relacionadas con la prueba de fluidez verbal, ya que es una tarea de producción libre en la que las personas originan respuestas de un modo abierto en un tiempo limitado, produciéndose una gran cantidad de datos que hay que organizar, analizar y cuantificar, y donde el carácter subjetivo de los evaluadores en la inclusión/exclusión de las categorías en el estudio tiene una gran relevancia (Belinchón, Igoa y Rivière, 2004). 
En conclusión, las personas con EA presentan un grave deterioro de la MS. En las personas sanas, si bien, con el paso de los años se incrementa la fluidez verbal, también se observa un leve declive. En este sentido, podría ser adecuado comprobar si, con otro tipo de tarea de evaluación de la misma, se producirían, efectivamente, los mismos resultados.

\section{REFERENCIAS}

Auriacombe, S., Fabrigoule, C., Lafont, S., JacqminGadda, H., y Dartigues, J.F. (2001). Letter and category fluency in normal elderly participants: A populationbased study. Aging, Neuropsychology and Cognition, 8, 98-108.

Barbarotto, R., Laiacona, M., Macchi, V., y Capitani, E. (2002). Picture reality decision, semantic categories and gender. A new set of pictures, with norms and an experimental study. Neuropsychologia, 40, 1637-1653.

Battig, W.F. y Montague, W.E. (1969). Category norms for verbal items in 56 categories: A replication and extension of the Connecticut category norms. Journal of Experimental Psychology Monographs, 80, 1-46.

Belinchón, M., Igoa, J.M., y Rivière, A. (2004). Psicología del lenguaje. Investigación y teoría ( $6^{\mathrm{a}} \mathrm{ed}$.). Trotta: Madrid.

Buiza, C. (2001). Evaluación y tratamiento de los trastornos del lenguaje. Matia Fundazioia. Recuperado de http://www.imsersomayores.csic.es/documentos/documentos/buiza-trastornosleguaje-01.pdf

Buriel, Y., Gramunt, N., Böhm, P., Rodés, E., y Peña-Casanova, J. (2004). Fluencia verbal. Estudio normativo piloto en una muestra española de adultos jóvenes (20 a 49 años). Neurología, 19, 153-159.

Burke, D.M., Mackay, D., Whorthey, J., y Wade, E. (1991). On the tip of the tongue: What causes word finding failures in young and old adults? Journal of Memory and Language, 30, 542-579.

Burke, D.M., Whorthey, J., y Martin, J. (1988). I'll never forget what's-her-name: Aging and tip of the tongue experiences in everyday life. En M.M. Gruneberg, P.E. Morris y R.N. Sykes (Eds.), Practical aspects of memory: Current research and issues (Vol. 2). Chichester: Wiley and Sons.

Camarazza, A. (1990). Cognitive neuropsychology and neurolinguistics: Advances in models of cognitive function and impairment. Hillsdale: Lawrence Erlbaum Associates.

Capitani, E., Laiacona, M. y Barbarotto, R. (1999). Gender affects word retrieval of certain categories in semantic fluency tasks. Cortex, 35, 273-278.
Cuetos, F., Rodríguez-Ferreiro, J., y Martínez, C. (2003). Alteraciones de memoria en los inicios de la enfermedad de Alzheimer. Revista Española de Neuropsicología, 5, 15-31.

De Vega, M. (2007). Introducción a la Psicología Cognitiva. Madrid: Alianza.

Díaz, C., y Peraita, H. (2008). Neuropsychological evaluation and cognitive evolution of a bilingual Alzheimer patient. Revista de Psicopatología y Psicología Clínica, 13, 219-228.

Díaz, C., Peraita, H. y Garriga, A.J. (2000). Problemas metodológicos al analizar datos de producción de ejemplares y de atributos en un estudio sobre deterioro semántico en enfermos de Alzheimer. Psicothema, 12, 192-195.

Diges, M. y Perpiñá, C. (2008). Psicopatología de la Memoria. En A. Belloch, B. Sandín y F. Ramos (Eds), Manual de Psicopatología (ed. rev, Vol. 1). Madrid: McGraw-Hill.

Fernández-Calvo, B., Contador, I., Serna, A., Meneces de Lucena, V., y Ramos, F. (2010). El efecto del formato de intervención individual o grupal en la estimulación cognitiva de pacientes con enfermedad de Alzheimer. Revista de Psicopatología y Psicología Clínica, 15, 125-131.

Galeote, M.A. y Peraita, H. (1999). Memoria semántica y fluidez verbal en demencias. Revista Española de Neuropsicología, 1, 3-17.

Gladsjo, J.A., Schuman, C.C., Evans, J.D., Peavy, G.M., Miller, S.W., y Heaton, R.K. (1999). Norms for letter and category fluency: demographic corrections for age, education, and ethnicity. Assessment, 6, 147-178.

Gonnerman, L.M., Andersen E.S., Devlin, J.T., Kempler, D., y Seidenberg, M.S. (1997). Doble dissociation of semantic categories in Alzheimer's disease, Brain and Language, 57, 254-279.

Gordon, H.W. y Lee, P.A. (1986). A relationship between gonadotropins and visuospatial function. Neuropsychologia, 24, 563-576.

Gutiérrez-Cabello, L., Aguilar-Alonso, A., Dávalos, A., y Pedraza, S. (2007). Rendimiento cognitivo y síntomas afectivos en la fase aguda y tres meses después de un ictus isquémico. Revista de Psicopatología y Psicología Clínica, 12, 177-187.

Henry, J.D., Crawford, J.R., y Phillips, L.H. (2004). Verbal fluency performance in dementia of the Alzheimer's type: a meta-analysis. Neuropsychologia 42, 12121222.

Hodges, J.R., Salmon, D.P., y Butters, N. (1991). The nature of naming deficit in Alzheimer's and Huntington's disease. Brain, 114, 1547-1558.

Huppert, F.A. (1991). Age-related changes in memory: Learning and remembering new information. En F. Boller y J. Grafman (Eds.), Handbook of Neuropsychology, 5 (pp. 123-147). Amsterdam: Elsevier. 
Koren, R., Kofman, O., y Berger, A. (2005). Analysis of word clustering in verbal fluency of school-aged children. Arch Clin Neuropsychol, 20, 1087-1104.

Lara-Useche, E., Pineda, D.A., Henao-Arboleda, E., Arboleda-Ramírez, A., Aguirre-Acevedo, D.C., y Lopera, F. (2006). Descripción del desempeño en memoria semántica en una muestra de la población antioqueña. Revista de Neurología, 42, 272-276.

Laws, K.R. (2004). Sex differences in lexical size across semantic categories. Personality and Individual Differences, 36, 23-32.

Marra, C., Ferraccioli, M., y Gainotti, G. (2007). Genderrelated dissociations of categorical fluency in normal subjects and in subjects with Alzheimer's disease. Neuropsychology, 21, 207-211.

Mervis, C.B. y Greco, C. (1984). Parts and early conceptual development: Comments on Tversky and Hemenway. Journal of Experimental Psychology: General, 113, 194-197.

Mervis, C.B., Catlin, J., y Rosch, E. (1975). Development of the structure of color categories. Developmental Psychology, 11, 54-60.

Miller, G.A. y Gildea P.M. (1987). How children learn words. Scientific American 257, 86-91.

Monsch, A.U., Bondi, M.W. Butters, N., Salmon, D.P., Katzman, R., y Thal, L.J. (1992). Comparisions of verbal fluency tasks in the detection of dementia of the Alzheimer type. Archives of Neurology, 49, 12531258.

Moreno, F.J. (2006). Una revisión de las principales tareas para evaluar el deterioro semántico en la enfermedad de Alzheimer. Acción Psicológica, 4, 57-68.

Moreno, F.J. y Cañamón, S. (2005). Presentación y resultados preliminares de la Batería Nombela (I). Un nuevo instrumento para evaluar el deterioro semántico categorial. Revista de Psicopatología y Psicología Clínica, 10, 205-219.

Moreno, I., Adrián, J.A., Buiza, J.J., y González, M. (2004). Disponibilidad y fluidez oral de palabras por categorías semánticas: un estudio prospectivo en adultos de más de 55 años con diferente nivel de escolarización. Revista de Logopedia, Foniatría y Audiología, 24, 106-118.

Moreno-Martínez, F.J. y Peraita, H. (2007). Un nuevo conjunto de ítems para las evaluación de la disociación ser vivo/ser no vivo con normas obtenidas de ancianos españoles. Psicológica, 28, 1-20.

Moreno-Martínez, F.J. y Peraita, H. (2007). Un nuevo conjunto de ítems para las evaluación de la disociación ser vivo/ser no vivo con normas obtenidas de ancianos españoles. Psicológica, 28, 1-20.

Muela, J.A., García-León, A., y Jiménez, M.D. (2007). Relación de la esquizotipia psicométrica con variables emocionales y socioambientales. Revista de Psicopatología y Psicología Clínica, 12, 199-212.
Neimark, E.D. (1974). Natural language concepts: additional evidence. Child Development, 45, 508-511.

Nelson, K. (1974). Variations in children's concepts by age and category. Child Development, 45, 577-584.

Ober, B.A., Dronkers, N.F., Koss, E., Delis, D.C., y Friedland, R.P. (1986). Retrieval from semantic memory in Alzheimer-type dementia. Journal of Clinical and Experimental Neuropsychology, 8, 75-92.

Peraita, H. y Sánchez-Bernardos, M.L. (1998). Evaluación del deterioro de diversos aspectos de la memoria semántica en pacientes de Alzheimer. Anales de Psicología, 14, 193-196.

Peraita, H., González Labra, M.J., Sánchez Bernardos, M.L., y Galeote, M.A. (2000). Batería de evaluación del deterioro de la memoria semántica en Alzheimer. Psicothema, 12, 192-200.

Pineda, D., Galeano, L.M., y Giraldo, Y. (1995). Prueba de memoria semántica con incremento asociativo. En D. Pineda y A. Ardila (eds), Neurología, evaluación clínica y psicometría (pp. 69-90). Prensa Creativa: Medellín.

Piñeiro, A., Morenza, L., Torres, R. y Sierra, C.E. (1999). Estudio normativo de veinte categorías semánticas en niños y adultos. Revista de Psicología General y Aplicada, 52, 147-157.

Posnansky, C.J. (1978). Category norms for verbal items in 25 categories for children in Grades 2-6. Behavior Research Methods \& Instrumentation, 10 , 819-832.

Puente, A. y Pogglioli, L. (1993). Categorías naturales: una medida de su estructura interna. Revista de Psicología General y Aplicada, 46, 387-396.

Ramírez, M., Ostrosky-Solís, F., Fernández, A. y ArdillaArdilla, A. (2005). Fluidez verbal semántica en hispanohablantes: un análisis comparativo. Revista de Neurología, 41, 463-468.

Román, F. y Sánchez, J.P. (1998). Cambios neuropsicológicos asociados al envejecimiento normal. Anales de Psicología, 14, 27-43.

Rosch, E. (1975). Cognitive Representations of semantic categories. Journal of Experimental Psychology: General, 104, 192-233.

Rosner, S.R. y Hayes, D.S. (1977). A developmental study of category ítem production. Child Development, 48, 1062-1065.

Salthouse, T.A. (1988). Effects of aging on verbal abilities: examination of the psychometric literature. En L.L. Light y D. Burke (Eds.), Language, Memory and Aging. Nueva York: Cambridge University Press.

Sauzeon, H., Lestage, P., Raboutet, C., N'Kaoua, B., y Claverie, B. (2004). Verbal fluency output in children aged 7-16 as a function of the production criterion: qualitative analysis of clustering, switching processes, and semantic network exploitation. Brain Language, 89, 192-202. 
Sebastián, M.V. y Elosúa, M.R. (2002). Tipo de material y olvido a corto plazo en pacientes Alzheimer. Psicothema, 14, 199-204.

Shafto, M.A., Burke, D.M., Stamatakis, E.A., Tamp, P.P. y Tyler, L.K. (2007). On the tip-of-the-tongue: Neural correlates of increased word-finding failures in normal aging. Journal of Cognitive Neuroscience, 19, 2060-2070.

Soto, M.P. (1981). Adquisición de términos y formación de conceptos: un estudio evolutivo. Tesis doctoral, Universidad Complutense de Madrid, Madrid

Soto, P., Sebastián, M.V., García, E. y del Amo, T. (1982). Categorización y datos normativos en España. Madrid: Cantoblanco [Las categorías y sus normas en castellano, 1994, Madrid, Visor].

Stumpf, H. (1995). Gender differences in performance on tests of cognitive abilities: Experimental design issues and empirical results. Psychological and psychobiological perspectives on sex differences in cognition: I. Theory and research. Learning and Individual Differences, $7,275-287$.

Tippett, L.J., Mcauliffe, S. y Farah, M. (1995). Preservation of categorical Knowledge in Alzheimer's disease: A computational account. Memory, 3, 519-533.
Torres, R., Piñeiro, A., Morenza, L. e Inguanzo, G. (2000). Estudio de seis categorías semánticas en niños pequeños. Revista Cubana de Psicología, 17, 106-113.

Tudela, P. (1985). Psicología Experimental. Madrid: UNED.

Uyeda, K.M. y Mandler, G. (1980). Prototipicality norms for 28 semantic categories. Behavior Research Methods and Instrumentation, 12, 587-595.

Valencia, N.J., Laserna, J.A., Pérez-García, M., Orozco, C., Minan, M., Garrido, C., et al. (2000). Influencia de la escolaridad y el sexo sobre la ejecucion en el FAS, nombrar animales y nombrar frutas. Psicología Conductual, 8, 283-295.

Wiederholt, W.C., Cahn, D., Butters, N.M., Salmon, D.P., Kritz-Silverstein, D. y Barrett-Connor, E. (1993). Effects of age, gender and education on selected neuropsychological tests in an elderly community cohort. Journal of the American Geriatrics Society, 41, 639647.

Wingfield, A., Aberdeen, J.S. y Stine, E.A. (1991). Word onset gating and linguistic context in spoken word and recognition by young and elderly adults. The Journals of Gerontology: Psychological Sciences, 46, 127-129. 